\title{
TENDENCIAS RECIENTES E ÍNDICES DE CAMBIO CLIMÁTICO DE LA TEMPERATURA Y LA PRECIPITACIÓN EN ANDORRA, PIRINEOS (1935-2008)
}

\author{
Recent trends and climate change indices of precipitation \\ and temperature in Andorra (1935-2000)
}

\author{
P. Esteban Vea ${ }^{1}$, M. Prohom Duran ${ }^{2} \&$ E. Aguilar $^{3}$ \\ 1 Centre d'Estudis de la Neu i de la Muntanya d'Andorra (CENMA-IEA). Av. Rocafort 21-23, \\ Edifici Molí3r pis, AD600 Sant Julià de Lòria (Andorra). pesteban.cenma@iea.ad \\ 2 Equip de Canvi Climátic-Àrea de Climatologia-Servei Meteorològic de Catalunya (SMC). \\ C. Berlín, 38-464a planta,08029 Barcelona.mprohom@meteo.cat \\ 3 Centre en Canvi Climàtic (C3)-Departament de Geografia, Universitat Rovira i Virgili \\ de Tarragona.Av. Catalunya, 35, 43002,Tarragona.enric.aguilar@urv.cat
}

ABSTRACT.- This paper shows the results obtained for the calculation of annual and seasonal trends of maximum and minimum temperature and precipitation from three climatic series of the Principality of Andorra (Pyrenees), and located between 1140 and the $1645 \mathrm{~m}$. The estimated trends have been obtained for the period 1935-2008 corresponding to the whole length of the series, and for the sub-period 1950-2008. From the same series, at a daily resolution, the indices of change defined by the ETCCDMI (Expert Team on Climate Change Detection Monitoring and Indices) have been also calculated.

The results represent a solid description of the Andorran climate dynamics over the past eight decades and provide insight into the variability and climate change experienced in the Pyrenean country, characterized by its high altitude. The results confirm the trends identified regionally by other agencies, both French and Spanish. This confirms the significant increase in air temperature for the annual mean maximum temperature $\left(0.13\right.$ to $0.15{ }^{\circ} \mathrm{C} /$ decade $)$, for the summer maximum temperature $\left(0.22{ }^{\circ} \mathrm{C} /\right.$ decade $)$, and the summer minimum temperature $\left(0.11^{\circ} \mathrm{C} /\right.$ decade). These trends are reinforced when the analysis focuses on the sub-period 1950-2008. For precipitation and for the period 1935-2008, only significant decreasing trends are obtained in the station Central and in summer (-5.4\%/decade), while this behavior is generalized and strengthened if we stick to the period 1950-2008. Finally, the results for the calculation of the ETCCDMI daily indices confirm and detail the results obtained at coarser resolution. Thus, the indices computed from the 


\section{P. ESTEBAN VEA, M. PROHOM DURAN \& E. AGUILAR}

temperature series show an increase in extreme warm days and a decrease of their opposites. For precipitation rates, significant trends were not found for the period 1935-2008, while several indices did show statistically significant decreasing values for the sub-period 1950-2008.

Keywords: Temperature, precipitation, homogeneity, trend analysis, Andorra.

RESUMEN.- En este trabajo se muestran los resultados obtenidos del cálculo de las tendencias anuales y estacionales de la temperatura máxima, temperatura mínima y la precipitación en el Principado de Andorra (Pirineos) a partir del análisis de tres series climáticas situadas entre los 1140 y los $1645 \mathrm{~m}$. Los cálculos de tendencia se han obtenido para el periodo 1935-2008 correspondiente a la longitud total de la series, así como para el sub-periodo 1950-2008. A partir de las mismas series a resolución diaria se han calculado los índices de cambio climático definidos por el ETCCDMI (Expert Teamfor Climate Change Detection Monitoring and Indices).

Los resultados confirman las tendencias detectadas regionalmente por otros organismos, tanto franceses como españoles. Así, se confirma el incremento significativo de la temperatura del aire para la temperatura máxima media anual $(0.13$ a $0.15{ }^{\circ} \mathrm{C} /$ década), para la máxima estival $\left(0,22^{\circ} \mathrm{C} /\right.$ década $)$, y la mínima estival $(0.11$ ${ }^{\circ} \mathrm{C} /$ década). Dichas tendencias al incremento térmico se refuerzan cuando el análisis se centra en el sub-periodo 1950-2008. En cuanto a la precipitación, se obtienen tendencias decrecientes y estadísticamente significativas para el periodo 1935-2008 en la estación de Central y en verano (-5.4\%/década), mientras que este comportamiento se generaliza y refuerza si nos ceñimos al periodo 1950-2008.

Finalmente, los resultados del cálculo de los índices diarios del ETCCDMI confirman y detallan lo ya obtenido a más baja resolución. Así, los indices computados a partir de las series de temperatura indican un claro incremento de extremos asociados a días cálidos y un descenso de sus opuestos. En cuanto a los índices de precipitación, no se detecta ninguno con tendencia significativa para el periodo 1935-2008, mientras que varios de ellos sí que presentan valores decrecientes con significación estadística para el sub-periodo 1950-2008.

Palabras clave: Temperatura, precipitación, homogeneidad, análisis de tendencia, Andorra.

\section{Introducción}

Las zonas de montaña son uno de los ambientes más frágiles del planeta (Diazet al., 2003). En este sentido es de mucha importancia avanzar en el conocimiento del impacto que está teniendo y que puede tener el cambio climático en los recursos hídricos, la biodiversidad o las actividades turísticas, y así favorecer su mejor conservación y gestión en estas zonas. 
Existen estudios que valoran, a partir del uso de proyecciones climáticas, la exposición al cambio climático que tendrán las zonas de montaña (NoguésBravo et al. 2007), pero no es tan frecuente la existencia de trabajos que aborden con detalle la evolución reciente del clima a partir de datos observados en estaciones situadas a altitudes medias y altas a causa de la dificultad de disponer de largas series de datos. Es destacado el ejemplo del macizo de los Alpes, donde desde hace años se trabaja en la recuperación y homogeneización de series climatológicas; es el llamado proyecto HISTALP (Böhm et al. 2009). Desde esta perspectiva, la situación es aun relativamente precaria en los Pirineos, sobre todo si nos referimos a emplazamientos situados en altitud.

Para avanzar en este sentido y en el ámbito pirenaico, el presente trabajo muestra los resultados del análisis de tendencias de las medias anuales y estacionales de la temperatura máxima y mínima del aire, así como de las acumulaciones anuales y estacionales de la precipitación para el Principado de Andorra y durante el periodo 1935-2008. Los datos, originales a resolución diaria y que se han revisado y corregido cuando ha sido necesario, también se han utilizado para calcular los índices de cambio climático sugeridos por el ETCCDMI (ExpertTeamforClimateChangeDetectionMonitoring and Indices).

En 2009 ya se realizó una primera aproximación al cálculo de tendencias de temperatura y precipitación a partir de la homogenización de estas series andorranas (Esteban et al. 2009). En el presente artículo se revisa y amplia lo obtenido anteriormente, aplicando el nuevo método de homogenización generado en 2011 por el proyecto COST-ES0601 (http://www.homogenisation.org/), además de aportar los resultados de índices diarios de cambio climático gracias a la corrección de las series diarias.

Los datos utilizados han sido las de tres estaciones meteorológicas instaladas y gestionadas por FHASA (Fuerzas Hidroeléctricas de Andorra, SA) y, desde 1988, por FEDA (Fuerzas Eléctricas de Andorra), que son: Central (Común de Encamp, 1.140 m), Engolasters (Comú de Escaldes-Engordany, $1.640 \mathrm{~m}$ ) y Ransol (Común de Canillo, $1.645 \mathrm{~m}$ ). En todas ellas se han hecho observaciones manuales diarias a las 8 de la mañana (hora local) hasta la última década, cuando progresivamente se ha pasado a la observación con sensores automáticos (Figura 1). Las tres series se presentan continuas desde el inicio de las observaciones en Octubre de 1934, con la excepción de lagunas puntuales. La disponibilidad de datos alcanza el $99 \%$ respecto al total teórico. 


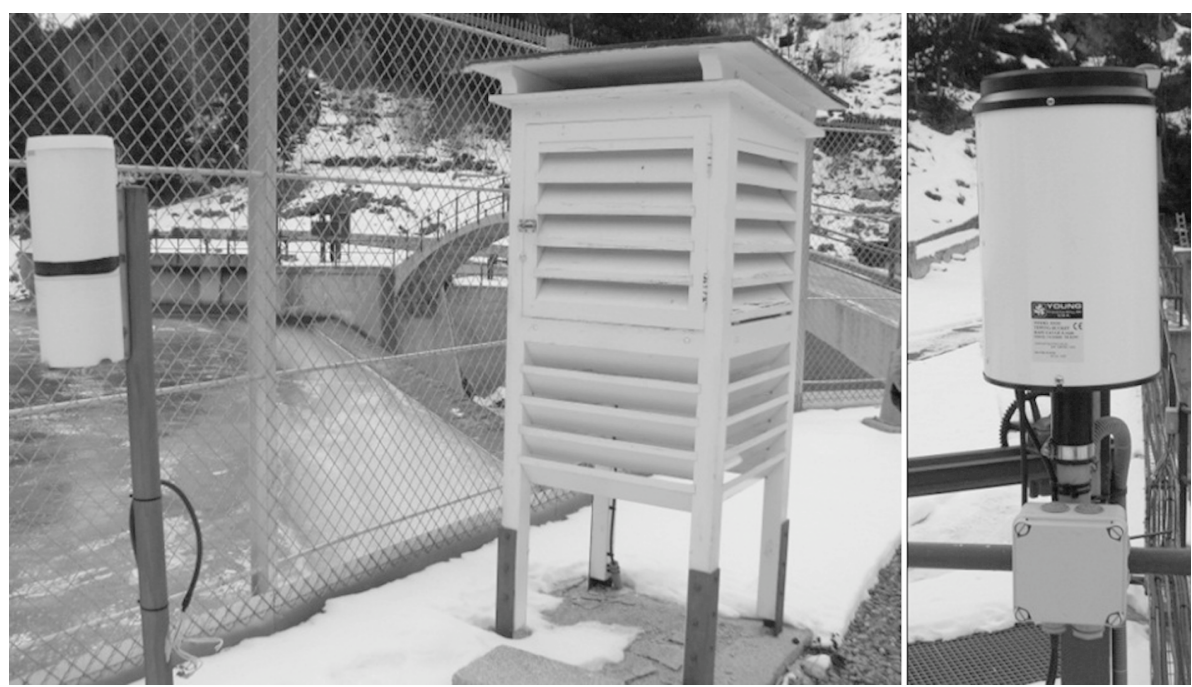

Figura 1. A la izquierda podemos ver la garita meteorológica y el pluviómetro manual de la estación meteorológica de Ransol (1645m). El pluviómetro manual tipo Hellmann ha sido sustituido por aparatos automáticos modernos como se observa a la derecha.

Figure 1. Ransol weather station (1645m). The manual Hellmann type rain gauge (left) has been replaced by modern automatic devices (right).

\section{Control de calidad y homogenización mensual y diaria}

Un paso previo ineludible antes del análisis de una serie climática es sin duda el estudio de su calidad y homogeneidad. El control de calidad consiste en un conjunto de pruebas jerarquizadas que tienen como objetivo detectar errores no sistemáticos y que pueden alterar la interpretación posterior de los datos. Estas pruebas se clasifican en los siguientes niveles y se han basado en las directrices de Brunet et al. (2008) y algunas mejoras realizadas por los autores:

- Identificación de fechas duplicadas.

- Grandes errores: valores de temperatura superiores a $50^{\circ} \mathrm{C}$ e inferiores a $-30^{\circ} \mathrm{C}$, o precipitación diaria superior a $500 \mathrm{~mm}$.

- Pruebas de consistencia interna: temperatura máxima igual o superior a la temperatura mínima, diferencias excesivas entre días consecutivos $\left(>20^{\circ} \mathrm{C}\right)$ y búsqueda de secuencias de valores idénticos consecutivos $(>4$, aplicado también a precipitación superior a 0 $\mathrm{mm})$. 
- Pruebas de tolerancia: identificación de valores fuera de rango, definidos como aquellos que superan 4 veces la desviación estándar y 3 veces el rango intercuartílico.

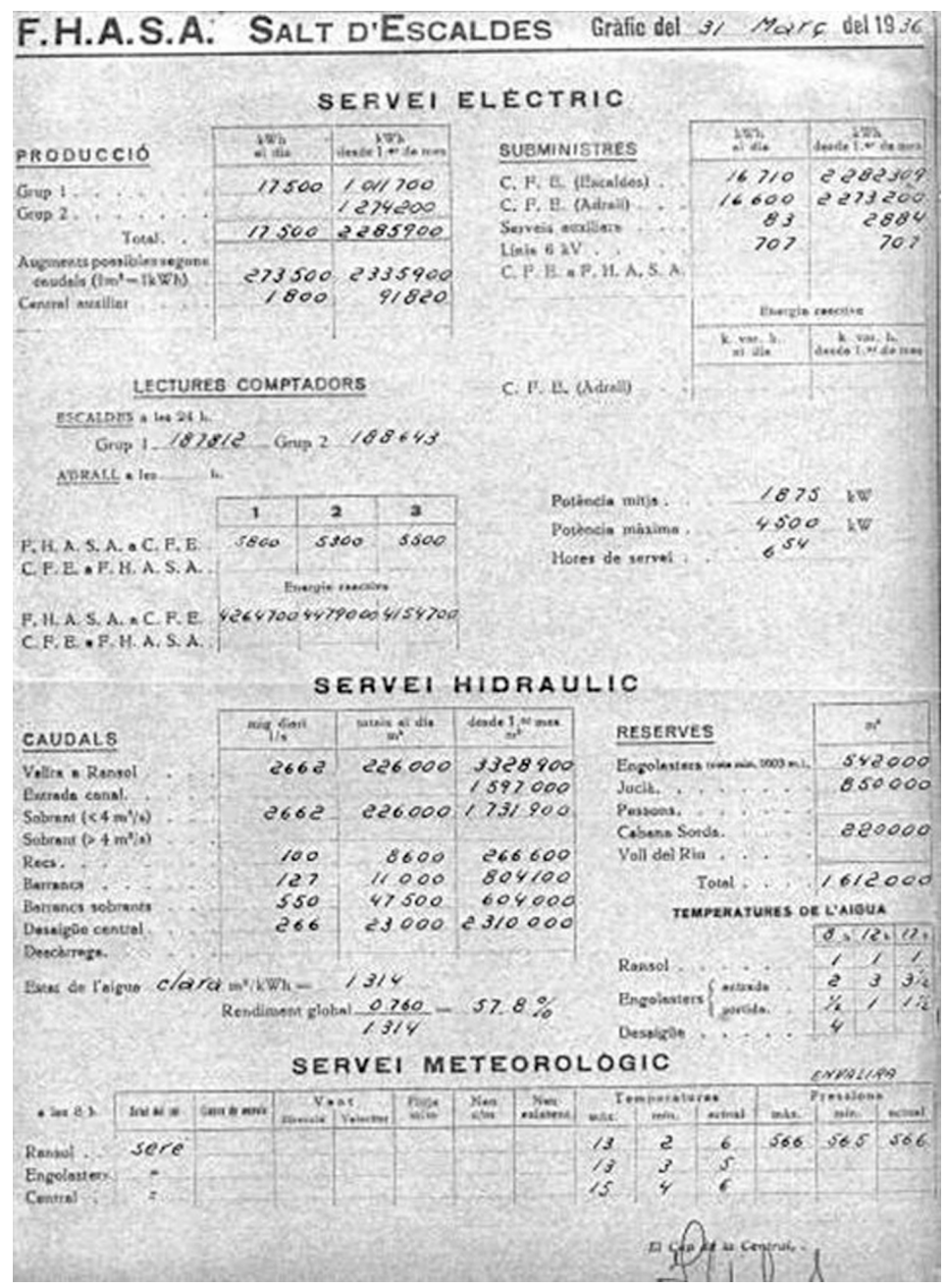

Figura 2. Ejemplo de las hojas originales consultables en el archivo de FEDA. En la parte inferior, se pueden ver los datos correspondientes a la observación meteorológica.

Figure 2. Example of the original sheet available at the FEDA archive. At the bottom, you can view the meteorological observations. 
Para el caso exclusivo de la precipitación se ha realizado un análisis adicional sobre los totales mensuales en una doble vía: identificación y validación de valores fuera de rango y discriminación entre meses sin observación y meses con precipitación nula. Fruto del análisis de calidad se detectan un conjunto de valores dudosos o erróneos que han sido corregidos, cuando esto ha sido posible, o bien eliminados.

En el caso que nos ocupa, la mayoría de los valores que se han identificado como sospechosos o erróneos van asociados al archivo y digitalización de los datos y, la mayor parte de ellos han sido resueltos mediante la consulta de la fuente original existente en el archivo de FEDA (figura 2).

\subsection{Análisis de homogeneidad mensual y anual}

Una vez asegurada la calidad de la información, es decir, eliminados los errores no sistemáticos, debe tratarse su homogeneidad. Una serie es homogénea cuando toda la variabilidad y el cambio presente en ella está relacionada únicamente con la deriva del clima y no es consecuencia de otros elementos externos y artificiales que la pueden haber perturbado (cambios de emplazamiento, de instrumental, de entorno, etc.) (Aguilar et al., 2003). Entre los métodos que analizan la homogeneidad de series climáticas, los más fiables son los métodos de homogeneización relativa, es decir, un conjunto de series coetáneas que comparten la misma señal climática se comparan para detectar y ajustar puntos de inhomogeneidad, permitiendo un correcto análisis posterior. Para este trabajo se han considerado las aportaciones de la recientemente terminada acción COST-ES0601 (Advances in Homogenisationmethods of climate series: anintegratedapproach, $H O M E$ ), que ha trabajado en la determinación de las metodologías más precisas, tanto para la detección como para el ajuste de inhomogeneidades (Venemaet al., 2011). El método resultante, HOMER -cuya aplicación en este trabajo es pionera-, se basa en el modelo descrito por Caussinus-Mestre (2004) que asume que cada uno de los valores de las series que forman el conjunto a homogenizar puede ser descompuesto en un efecto climático -común a todas ellas- y un efecto de estación, constante en caso de serie homogénea, variable en el tiempo en caso de serie inhomogénea. El antecedente directo de HOMER llamado "PRODIGE"- comparaba las series de forma emparejada (pairwise) con el fin de detectar el número y posición (Caussinus\&Lyazhri, 1997) de los puntos de cambio. HOMER añade -gracias a la incorporación del algoritmo descrito por Pickard et al (2011)- la posibilidad de realizar la detección simultánea en el conjunto de series. Ambas técnicas se superponen y se ayudan -en el caso de la temperatura- del método de detección ACMANT (Domonkos, 2011). Finalmente, 
la consulta del metadato permite introducir/eliminar puntos de cambio que aparecen como artificio estadístico. El ajuste se realiza mediante el uso de los coeficientes del modelo utilizado en la detección conjunta.

Para el caso andorrano, se han utilizado 30 series climáticas de referencia con datos de temperatura máxima y temperatura mínima mensual repartidas a ambas vertientes de los Pirineos. Para cada serie candidata se han escogido un mínimo de 10 series de referencia, con una correlación mínima de r=0.7, calculada con las series de primera diferencia. Para el caso de la precipitación y fruto de la buena calidad de las series, se ha constatado la ausencia de puntos de cambio en Ransol y Central. No ha sido así en Engolasters, por lo que la estación ha sido descartada.

\subsection{Homogeneidad diaria}

Para el análisis de los índices de extremos climáticos es necesario utilizar series climáticas a resolución diaria y datos de calidad y homogéneos. La homogeneización de series diarias es mucho más problemática que la realizada a escalas mensual o anual, especialmente por las dificultades de realizar ajustes a esta escala temporal más fina. Los modelos de regresión múltiple en los que intervienen otros parámetros como la insolación, la velocidad y dirección del viento y la existencia de medidas paralelas son los métodos de mayor fiabilidad para realizar el ajuste, pero la disponibilidad de estos elementos es muy escasa. Existen también otros métodos basados en la comparación de series altamente correlacionadas cuyos puntos de cambio no se sobreponen, permitiendo la modelización entre ambas y la derivación de factores de ajuste (Della-Marta y Wanner, 2007; Mestreet al., 2011). Las series de Andorra no cumplen con los requisitos necesarios (Klugitschet al., 2009), por ello, se hace necesario el uso de otros métodos menos exigentes, tales como el propuesto por Vincentet al. (2002) que se basa en la interpolación de los coeficientes de corrección obtenidos de las correcciones mensuales. Esta metodología permite una aproximación razonable, especialmente a la hora de computar índices, puesto que la frecuencia de extremos se corrige correctamente, aunque no así su posición.

Las series diarias de temperatura de Andorra han sido homogeneizadas siguiendo este procedimiento, usando los factores de corrección obtenidos a partir de la homogeneización mensual propuesta por el método HOMER. Para el caso de las series diarias de precipitación no se ha realizado ningún ajuste al no detectarse puntos de discontinuidad a resoluciones mensual o anual. 


\subsection{Cálculo de tendencias e indices climáticos}

Finalmente, las tendencias de la temperatura y la precipitación para los periodos 1935-2008 y 1950-2008 se han calculado mediante la aproximación tradicional por mínimos cuadrados. Dichas tendencias obtenidas, que se presentan en grados centígrados/década para la temperatura y porcentaje de cambio / década para la precipitación, van acompañadas de la correspondiente significación estadística, con un nivel de confianza (o significación) del 95\% según el test de Mann-Kendall.

Para la obtención de les índices climáticos se ha seguido la pauta definida por el Grupo de Expertos en Índices para la Detección del Cambio Climático (Expert Teamon Climate Change Detection and Indices, ETCCDI) (Peterson et al. 2001). Para ello se ha utilizado el software de distribución libre R-ClimDex, en el cual se definen 27 índices para series diarias de temperatura máxima, temperatura mínima y precipitación.

\section{Tendencias anuales y estacionales}

\subsection{Temperatura del aire}

Los resultados obtenidos para el período 1935-2008 muestran que la temperatura máxima media anual (Tabla 1) ha aumentado en las tres estaciones de forma significativa, mientras la temperatura mínima media anual (Tabla 2) presenta una pendiente positiva sin significación.

Tabla 1. Tendencias obtenidas para la temperatura media de las máximas en las tres estaciones andorranas para el periodo 1935-2008. Los valores en negrita y con asterisco son estadísticamente significativos en un nivel de confianza del 95\%, según el test de Mann-Kendall.

Table 1. Trends obtained for the average maximum temperature in the three Andorran stations for the period 1935-2008. Values in bold with an asterisk are statistically significant at a confidence level of 95\%, according to the Mann-Kendall.

\begin{tabular}{|ccc|}
\hline \multicolumn{3}{|c|}{ TEMPERATURA MÁXIMA (CENTRAL/ENGOLASTERS/RANSOL) } \\
\hline 1935-2008 & TENDENCIA DECENAL & Intervalos de confianza \\
& $\left({ }^{*} /\right.$ década) & $\left({ }^{\circ}\right.$ C/década) \\
Anual & $0.15^{*} / 0.14 * / 0.13^{*}$ & {$[0.07 / 0.22] /[0.05 / 0.22] /[0.05 / 0.21]$} \\
Invierno & $0.15^{*} / 0.13 / 0.14^{*}$ & {$[0.02 / 0.29] /[-0.01 / 0.26] /[0.01 / 0.26]$} \\
Primavera & $0.12 / 0.11 / 0.11$ & {$[-0.01 / 0.26] /[-0.02 / 0.24] /[-0.03 / 0.25]$} \\
Verano & $0.22 * / 0.22 * / 0.22 *$ & {$[0.10 / 0.34] /[0.09 / 0.35] /[0.08 / 0.35]$} \\
Otoño & $0.09 / 0.07 / 0.07$ & {$[-0.03 / 0.21] /[-0.08 / 0.22] /[-0.06 / 0.19]$} \\
\hline
\end{tabular}


TENDENCIAS RECIENTES E ÍNDICES DE CAMBIO CLIMÁTICO DE LA TEMPERATURA

Tabla 2. Tendencias obtenidas para la temperatura media de las mínimas en las tres estaciones andorranas para el periodo 1935-2008. Los valores en negrita y con asterisco son estadísticamen te significativos en un nivel de confianza del 95\%, según el test de Mann-Kendall.

Table 2. Trends obtained for the average minimum temperature in the three Andorran stations for the period 1935-2008. Values in bold with an asterisk are statistically significant at a confidence level of $95 \%$, according to the Mann-Kendall.

\begin{tabular}{|ccc|}
\hline \multicolumn{3}{|c|}{ TEMPERATURA MÍNIMA (CENTRAL/ENGOLASTERS/RANSOL) } \\
\hline 1935-2008 & $\begin{array}{c}\text { TENDENCIA DECENAL } \\
\text { (o-C/década) }\end{array}$ & $\begin{array}{c}\text { Intervalos de confianza } \\
\text { (ㅇ/década) }\end{array}$ \\
Anual & $0.05 / 0.05 / 0.05$ & {$[-0.07 / 0.11] /[-0.02 / 0.12] /[-0.01 / 0.11]$} \\
Invierno & $0.10 / 0.09 / 0.07$ & {$[-0.00 / 0.21] /[-0.04 / 0.21] /[-0.05 / 0.18]$} \\
Primavera & $0.02 /-0.04 /-0.00$ & {$[-0.07 / 0.11] /[-0.15 / 0.07] /[-0.09 / 0.10]$} \\
Verano & $0.11^{*} / 0.11^{*} / 0.11^{*}$ & {$[0.03 / 0.20] /[0.01 / 0.21] /[0.03 / 0.19]$} \\
Otoño & $-0.03 / 0.04 / 0.01$ & {$[-0.12 / 0.07] /[-0.07 / 0.15] /[-0.08 / 0.10]$} \\
\hline
\end{tabular}

La tendencia de la temperatura máxima oscila entre 0.13 y $0.15^{\circ} \mathrm{C} /$ década de incremento anual, siendo significativo para todos los observatorios. Estacionalmente, los valores apuntan hacia el incremento, pero sólo en verano $\left(0.22^{\circ} \mathrm{C} /\right.$ década $)$ presenta tendencias más marcadas y estadísticamente significativas de forma unánime, mientras que en invierno lo hace para dos de las series (0.14 y $0.15^{\circ} \mathrm{C} /$ década en Ransol y Central, respectivamente).

En cuanto a las mínimas, un incremento de $0.05^{\circ} \mathrm{C} /$ década se obtiene para las tres estaciones andorranas, aunque este resultado no es significativo estadísticamente. Así, aunque no podemos pues confirmar esta tendencia al incremento, sí queremos apuntar la posibilidad de que esta falta de significación sea consecuencia de la elevada variabilidad invernal. Estacionalmente, nos encontramos con la misma situación que con las medias anuales de las mínimas. Así, se obtienen tendencias de incremento en todos los casos, pero sólo significativas estadísticamente para el verano $\left(0.11^{\circ} \mathrm{C} /\right.$ década $)$.

Por otro lado, si se observa el gráfico de evolución de la anomalía de temperatura media anual (figura 3) se puede afirmar que en general hay una acumulación de años cálidos especialmente en las últimas décadas del período de estudio, destacando 1994, 1997 y 2006.

Finalmente, se ha centrado el análisis en un período más reciente, y que se inicia en 1950 (tablas 3 y 4), al ser este un período común en muchos estudios. Los resultados reafirman los obtenidos para la globalidad del período disponible, incrementando la magnitud de los valores de tendencia y extendiendo la significación estadística. En este sentido, es interesante hacer notar que para las temperaturas máximas, solamente el otoño muestra tendencias 


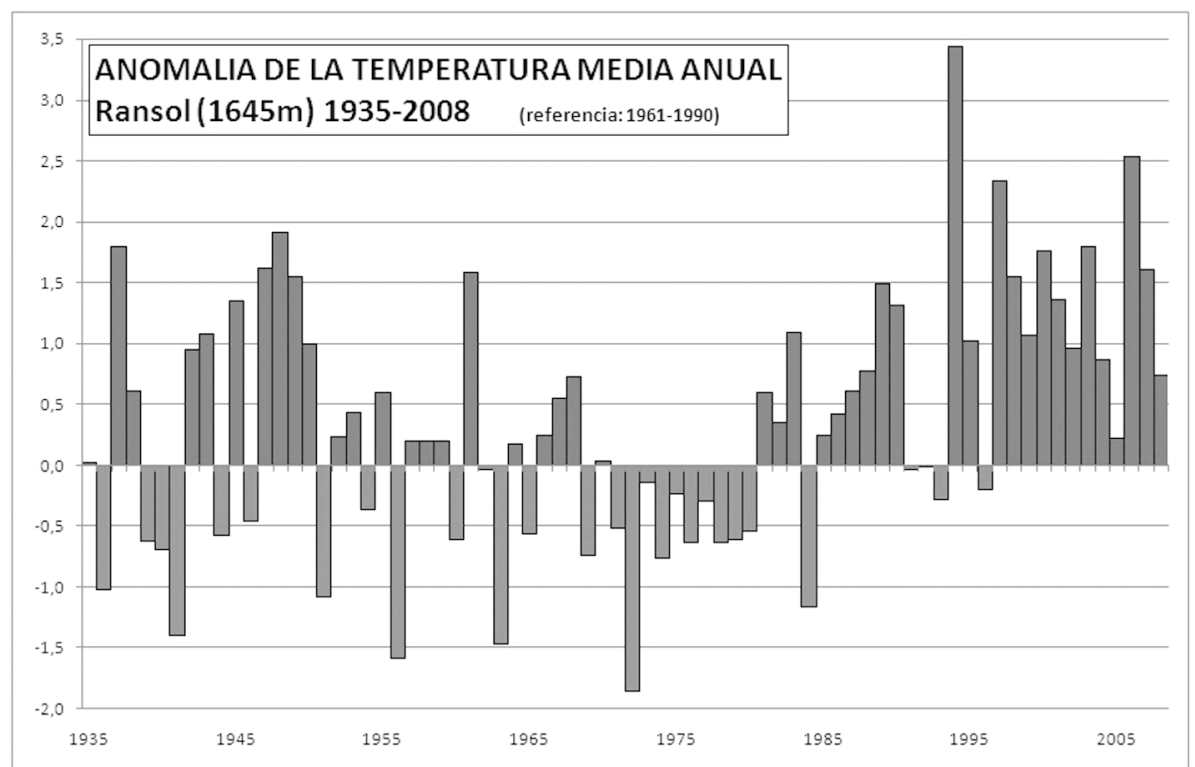

Figura 3. Anomalía de la temperatura media anual respecto al periodo 1961-1990 para la estación meteorológica andorrana de Ransol. Destaca el año 1994, donde la masa de aire asociada a los grandes incendios en Cataluña y con vientos del sur afectaron al Principado de Andorra.

Figure 3. Anomaly of the yearly temperature for the period 1961-1990 and the Ransol weather station. Highlights 1994, where the air mass associated with large forest fires in Catalonia affected the Principality of Andorra due to southern winds.

no significativas. De nuevo, el verano se muestra como la estación del año más afectada por el calentamiento térmico $\left(0.38\right.$ a $0.44{ }^{\circ} \mathrm{C} /$ década para las máximas y 0.16 a $0.21^{\circ} \mathrm{C} /$ década para las mínimas).

\subsection{Precipitación}

La evaluación de la calidad de las series y de su homogeneidad ha hecho relevante el alto grado de calidad de las observaciones de precipitación en dos de las estaciones de FEDA. Así, en las series de Central y Ransol no se han encontrado puntos de ruptura significativos que requieran el ajuste, aspecto especialmente problemático en series de precipitación diarias. Por ello, a diferencia de la temperatura, se ha optado por tomar los datos una vez pasado el control de calidad y obtener las tendencias anuales y estacionales, teniendo en cuenta las características inherentes unidas a esta variable 
TENDENCIAS RECIENTES E ÍNDICES DE CAMBIO CLIMÁTICO DE LA TEMPERATURA

Tabla 3. Tendencias obtenidas para la temperatura media de las máximas en las tres estaciones andorranas para el periodo 1950-2008. Los valores en negrita y con asterisco son estadísticamente significativos en un nivel de confianza del 95\%, según el test de Mann-Kendall.

Table 3. Trends obtained for the average maximum temperature in the three Andorran stations for the period 1950-2008. Values in bold with an asterisk are statistically significant at a confidence level of 95\%, according to the Mann-Kendall.

\begin{tabular}{|c|c|c|}
\hline \multicolumn{3}{|c|}{ TEMPERATURA MÁXIMA (CENTRAL/ENGOLASTERS/RANSOL) } \\
\hline 1950-2008 & $\begin{array}{l}\text { TENDENCIA DECENAL } \\
\text { (ํ/década) }\end{array}$ & $\begin{array}{c}\text { Intervalos de confianza } \\
\text { (으/década) }\end{array}$ \\
\hline Anual & $0.24 * / 0.26 * / 0.25 *$ & {$[0.14 / 0.34] /[0.17 / 0.38] /[0.15 / 0.37]$} \\
\hline Invierno & $0.23 * / 0.29 * / 0.25 *$ & {$[0.05 / 0.41] /[0.12 / 0.47] /[0.08 / 0.42]$} \\
\hline Primavera & $0.20 * / 0.23 * / 0.23 *$ & {$[0.01 / 0.38] /[0.04 / 0.41] /[0.04 / 0.42]$} \\
\hline Verano & $0.38 * / 0.44 * / 0.42 *$ & {$[0.21 / 0.55] /[0.27 / 0.61] /[0.23 / 0.60]$} \\
\hline Otoño & $0.16 / 0.18 / 0.13$ & {$[-0.01 / 0.34] /[-0.02 / 0.38] /[-0.05 / 0.31]$} \\
\hline
\end{tabular}

Tabla 4. Tendencias obtenidas para la temperatura media de las mínimas en las tres estaciones andorranas para el periodo 1950-2008. Los valores en negrita y con asterisco son estadísticamente significativos en un nivel de confianza del 95\%, según el test de Mann-Kendall. Table 4. Trends obtained for the average minimum temperature in the three Andorran stations for the period 1950-2008. Values in bold with an asterisk are statistically significant at a confidence level of 95\%, according to the Mann-Kendall.

\begin{tabular}{|ccc|}
\hline \multicolumn{3}{|c|}{ TEMPERATURA MÍNIMA (CENTRAL/ENGOLASTERS/RANSOL) } \\
\hline 1950-2008 & TENDENCIA DECENAL & Intervalos de confianza \\
& $($ oC $/$ década) & (óC/década) \\
Anual & $0.11^{*} / 0.12^{*} / 0.11^{*}$ & {$[0.03 / 0.20] /[0.03 / 0.21] /[0.03 / 0.19]$} \\
Invierno & $0.14 / 0.13 / 0.18^{*}$ & {$[-0.02 / 0.30] /[-0.03 / 0.30] /[0.02 / 0.33]$} \\
Primavera & $0.08 / 0.04 / 0.09$ & {$[-0.05 / 0.20] /[-0.12 / 0.19] /[-0.03 / 0.21]$} \\
Verano & $0.21 * / 0.25 * / 0.16^{*}$ & {$[0.09 / 0.33] /[0.11 / 0.38] /[0.06 / 0.26]$} \\
Otoño & $0.04 / 0.07 / 0.03$ & {$[-0.08 / 0.17] /[-0.08 / 0.23] /[-0.09 / 0.15]$} \\
\hline
\end{tabular}

(mayor variabilidad temporal y espacial y mayor dependencia de episodios puntuales). En la tabla 5 se presentan los resultados del periodo 1935-2008 en porcentaje / década, es decir, variación de la precipitación en tanto por ciento para un período de 10 años, y con la significación estadística con un nivel de confianza del 95\% según el test (o prueba) de Mann-Kendall.

Los resultados de Ransol y Central confirman la falta de tendencias significativas en cuanto a la precipitación anual y estacional en Andorra durante el periodo 1935-2008, con la única excepción del verano en la segunda de las series. Esto indica que en general no se podría hablar todavía de ningún 
incremento o descenso significativo en esta variable para el periodo de estudio, aunque todo apunte en la dirección que muestran los resultados para el periodo 1950-2008. Así, para la segunda mitad de siglo XX e inicios del actual la tendencia anual y la correspondiente al verano ya muestran claramente un signo decreciente y estadísticamente significativo (tabla 6). No es el caso para invierno, primavera y otoño, que a pesar de mostrar también un patrón decreciente, no pueden considerarse como tendencias sólidas.

Tabla 5. Tendencias obtenidas para la precipitación acumulada en dos estaciones andorranas para el periodo 1935-2008. Los valores en negrita y con asterisco són estadísticamente significativos en un nivel de confianza del $95 \%$, según el test de Mann-Kendall.

Table 5. Trends obtained for the accumulated rainfall at two Andorran stations for the period 19352008. Values in bold with an asterisk are statistically significant at a confidence level of 95\%, according to the Mann-Kendall.

\begin{tabular}{|ccc|}
\hline \multicolumn{3}{|c|}{ PRECIPITACION (CENTRAL/RANSOL) } \\
\hline 1935-2008 & $\begin{array}{c}\text { TENDENCIA DECENAL } \\
\text { (\%/década) }\end{array}$ & $\begin{array}{c}\text { Intervalos de confianza } \\
\text { (\%/década) }\end{array}$ \\
Anual & $-1.8 /-0.8$ & {$[-4.0 / 0.5] /[-2.8 / 1.2]$} \\
Invierno & $-1.2 /-1.3$ & {$[-6.9 / 4.5] /[-6.2 / 3.5]$} \\
Primavera & $0.5 / 0.8$ & {$[-2.9 / 3.9] /[-2.3 / 4.0]$} \\
Verano & $-5.4 * /-2.9$ & {$[-9.0 /-1.8] /[-6.2 / 0.4]$} \\
Otoño & $0.2 / 0.8$ & {$[-4.2 / 4.5] /[-3.3 / 4.9]$} \\
\hline
\end{tabular}

Tabla 6. Tendencias obtenidas para la precipitación acumulada en dos estaciones andorranas para el periodo 1950-2008. Los valores en negrita y con asterisco són estadísticamente significativos en un nivel de confianza del 95\%, según el test de Mann-Kendall.

Table 6. Trends obtained for the accumulated rainfall at two Andorran stations for the period 19502008. Values in bold with an asterisk are statistically significant at a confidence level of 95\%, according to the Mann-Kendall.

\begin{tabular}{|ccc|}
\hline \multicolumn{3}{|c|}{ PRECIPITACION (CENTRAL /RANSOL) } \\
\hline 1950-2008 & $\begin{array}{c}\text { TENDENCIA DECENAL } \\
\text { (\%/década) }\end{array}$ & $\begin{array}{c}\text { Intervalos de confianza } \\
\text { (\%/década) }\end{array}$ \\
Anual & $-4.1^{*} /-4.0^{*}$ & {$[-7.3 /-0.8] /[-6.6 /-1.8]$} \\
Invierno & $-4.3 /-4.5$ & {$[-12.1 / 3.6] /[-5.6 / 4.5]$} \\
Primavera & $-0.6 /-1.8$ & {$[-5.6 / 4.5] /[-6.2 / 2.7]$} \\
Verano & $-9.3 * /-7.2^{*}$ & {$[-14.6 /-4.1] /[-11.8 /-2.7]$} \\
Otoño & $-0.7 /-1.9$ & {$[-6.9 / 5.5] /[-7.6 / 3.9]$} \\
\hline
\end{tabular}


Igual que en todo estudio regional, los resultados de la precipitación se deben tomar con más cautela por la variabilidad espacial y temporal intrínseca a dicha variable. Más aún en este caso, ya que provienen de solamente dos estaciones y se relacionan con un entorno geográfico en el que parte de la precipitación se registra en forma sólida y, por tanto, se convierte en equivalente líquido. Este y otros procesos pueden introducir discontinuidades en las frecuencias de los valores bajos de precipitación que no se han investigado y que habrá que analizar con más profundidad para confirmar los resultados preliminares obtenidos hasta ahora.

\section{Cálculo de índices de cambio climático}

Gracias a la disponibilidad de los datos diarios continuos, de calidad óptima y homogeneizados según el método de Vincentet al. (2002), es posible realizar un análisis del comportamiento y tendencia de los eventos climáticos extremos, que tanto interés despiertan entre la comunidad científica, por sus evidentes impactos sobre nuestra sociedad (Klein Tank, 2006).

Los resultados muestran un comportamiento acorde con lo que sucede en el entorno andorrano. Así, los índices computados a partir de las series de temperatura indican un claro incremento de extremos asociados a días cálidos y un descenso de sus opuestos. La tabla 7 recoge los 20 índices de temperatura calculados para todo el período disponible (1935-2008) y para las tres series andorranas (los 16 definidos por el ETCCDI más otros cuatro definidos para cada serie, en respuesta a su especificidad y localización altitudinal, y los 11 índices obtenidos para las series de precipitación de Ransol y Central).

Entre los índices de temperatura cuatro presentan incrementos significativos en las tres series analizadas, y todos asociados a las temperaturas máximas: su25 (días de verano, o número de días en que la temperatura máxima supera $\operatorname{los} 25^{\circ} \mathrm{C}$ ), Txx (valor máximo anual de la temperatura máxima), Tx90p (días cálidos, o porcentaje de días en los que la temperatura máxima supera el umbral del percentil 90) y otros tantos índices de días de verano calculados utilizando otros umbrales para cada serie. También se aprecian otros comportamientos de interés y asociados al menos a dos de las tres series y que van más allá de la tendencia de la temperatura máxima. Así, en Ransol y Central se detecta un descenso en los días de helada (temperatura mínima inferior a les $0^{\circ} \mathrm{C}$ o inferiores a otros umbrales más exigentes) y en el número de noches frías (o porcentaje de días en los que la temperatura mínima es inferior al percentil 10), mientras que se aprecia un incremento en la tendencia del valor mínimo anual de la temperatura mínima (o tnn), en el número de rachas cálidas (es 


\section{P. ESTEBAN VEA, M. PROHOM DURAN \& E. AGUILAR}

Tabla 7. Resultados del cálculo de los índices del ETCCDMI con el programa RClimdex y para el periodo 1935-2008. Sombreado rojo indica incremento estadísticamente significativo, y sombreado azul descenso estadísticamente significativo, en ambos casos según el valor de $\mathrm{p}(\mathrm{p}<0.05)$. Los índices su 24/26.2/28.1, id -2.1/0.9/1.5, tr 8.3/13.1/12.4 y fd -10.6 / -7.3 / -5.9 se han calculado, respectivamente, con el valor medio de las máximas más altas para el mes más cálido (su), con valor medio de las máximas más baja para el mes más frio (id), con el valor medio de las mínimas más altas del mes más cálido (tr), y el valor medio de las mínimas más bajas del mes más frio (fd) según Raso (1999).

Table 7. Results for the ETCCDMI indices calculated with the software RClimDex and for the period 19352008. Shading indicates statistically significant increase/decrease (red/blue) for a $p$-value $<0.05$ in both cases. The rates su 24/26.2/28.1, id -2.1/0.9/1.5, $\operatorname{tr} 8.3 / 13.1 / 12.4$ and $\mathrm{fd}-10.6 /-7.3 /-5.9$ are calculated, respectively, with the average of the highest maximum temperatures for the warmest month (su) with the average of the lower maximum temperatures for the coldest month (id), with the average of the highest minimum temperatures in the warmest month (tr) and the average of the lower minimum temperatures for the coldest month (fd) calculated in Raso (1999).

\begin{tabular}{|c|c|c|c|c|c|c|c|c|c|}
\hline \multirow{2}{*}{$\begin{array}{c}\text { 1935-2008 } \\
\text { Indices }\end{array}$} & \multicolumn{3}{|c|}{ RANSOL $(1645 \mathrm{~m})$} & \multicolumn{3}{|c|}{ ENGOLASTERS $(1640 \mathrm{~m})$} & \multicolumn{3}{|c|}{ CENTRAL $(1145 \mathrm{~m})$} \\
\hline & Pend. & $\begin{array}{l}\text { Desv. } \\
\text { Pend. }\end{array}$ & P_Valor & Pend. & $\begin{array}{l}\text { Desv. } \\
\text { Pend. }\end{array}$ & P_Valor & Pend. & $\begin{array}{l}\text { Desv. } \\
\text { Pend. }\end{array}$ & P_Valor \\
\hline su25 & 0.146 & 0.043 & 0.001 & 0.141 & 0.037 & 0 & 0.146 & 0.064 & 0.026 \\
\hline ido & -0.033 & 0.05 & 0.517 & -0.041 & 0.039 & 0.297 & -0.059 & 0.019 & 0.003 \\
\hline $\operatorname{tr} 20$ & 0 & 0 & $\mathrm{NaN}$ & 0 & 0 & $\mathrm{NaN}$ & -0.004 & 0.002 & 0.041 \\
\hline $\mathrm{fdo}$ & -0.409 & 0.089 & 0 & -0.175 & 0.112 & 0.121 & -0.203 & 0.082 & 0.015 \\
\hline su $24 / 26.2 / 28.1$ & 0.213 & 0.053 & 0 & 0.07 & 0.028 & 0.014 & 0.186 & 0.043 & 0 \\
\hline id $-2.1 / 0.9 / 1.5$ & -0.059 & 0.033 & 0.08 & -0.046 & 0.046 & 0.327 & -0.087 & 0.032 & 0.008 \\
\hline tr $8.3 / 13.1 / 12.4$ & 0.024 & 0.056 & 0.671 & 0.148 & 0.049 & 0.003 & 0.12 & 0.067 & 0.077 \\
\hline $\mathrm{fd}-10.6 /-7.3 /-5.9$ & -0.047 & 0.038 & 0.221 & -0.057 & 0.038 & 0.14 & -0.094 & 0.037 & 0.013 \\
\hline gsl & 0.145 & 0.15 & 0.334 & 0.122 & 0.195 & 0.532 & -0.017 & 0.156 & 0.912 \\
\hline txx & 0.022 & 0.01 & 0.026 & 0.029 & 0.01 & 0.004 & 0.027 & 0.009 & 0.002 \\
\hline txn & 0.011 & 0.014 & 0.459 & 0.005 & 0.015 & 0.76 & 0.027 & 0.013 & 0.047 \\
\hline $\operatorname{tn} x$ & 0.017 & 0.009 & 0.063 & 0.021 & 0.008 & 0.008 & -0.009 & 0.008 & 0.244 \\
\hline $\operatorname{tnn}$ & 0.036 & 0.013 & 0.008 & 0.014 & 0.014 & 0.307 & 0.05 & 0.011 & 0 \\
\hline tx10p & -0.004 & 0.016 & 0.796 & -0.02 & 0.016 & 0.232 & -0.027 & 0.017 & 0.118 \\
\hline$t \times 90 p$ & 0.115 & 0.023 & 0 & 0.07 & 0.024 & 0.005 & 0.114 & 0.021 & 0 \\
\hline $\operatorname{tn} 10 p$ & -0.052 & 0.018 & 0.004 & -0.007 & 0.018 & 0.696 & -0.035 & 0.018 & 0.058 \\
\hline $\operatorname{tn} 90 p$ & 0.036 & 0.026 & 0.157 & 0.076 & 0.026 & 0.005 & 0.061 & 0.032 & 0.06 \\
\hline wsdi & 0.134 & 0.055 & 0.016 & 0.044 & 0.047 & 0.349 & 0.11 & 0.047 & 0.021 \\
\hline csdi & -0.044 & 0.027 & 0.105 & 0.031 & 0.028 & 0.285 & 0 & 0.025 & 0.994 \\
\hline dtr & 0.007 & 0.003 & 0.007 & 0.004 & 0.003 & 0.188 & 0.01 & 0.002 & 0 \\
\hline rx1day & 0.066 & 0.106 & 0.536 & & & & -0.029 & 0.109 & 0.79 \\
\hline rx5 day & -0.143 & 0.18 & 0.428 & & & & -0.4 & 0.201 & 0.051 \\
\hline sdii & 0.005 & 0.006 & 0.353 & & & & -0.005 & 0.007 & 0.455 \\
\hline $\mathrm{r} 10 \mathrm{~mm}$ & -0.052 & 0.038 & 0.171 & & & & -0.068 & 0.038 & 0.081 \\
\hline $\mathrm{r} 20 \mathrm{~mm}$ & -0.017 & 0.025 & 0.489 & & & & -0.032 & 0.02 & 0.111 \\
\hline $\mathrm{r} 50 \mathrm{~mm}$ & -0.001 & 0.006 & 0.879 & & & & -0.002 & 0.007 & 0.726 \\
\hline edd & -0.025 & 0.049 & 0.605 & & & & 0.023 & 0.051 & 0.662 \\
\hline owd & -0.019 & 0.01 & 0.06 & & & & -0.013 & 0.007 & 0.078 \\
\hline$r 95 p$ & 0.08 & 0.681 & 0.906 & & & & 0.187 & 0.621 & 0.764 \\
\hline $\mathrm{r} 99 \mathrm{p}$ & 0.002 & 0.385 & 0.997 & & & & -0.145 & 0.361 & 0.688 \\
\hline preptot & -0.991 & 1.044 & 0.346 & & & & -1.419 & 1.016 & 0.167 \\
\hline
\end{tabular}




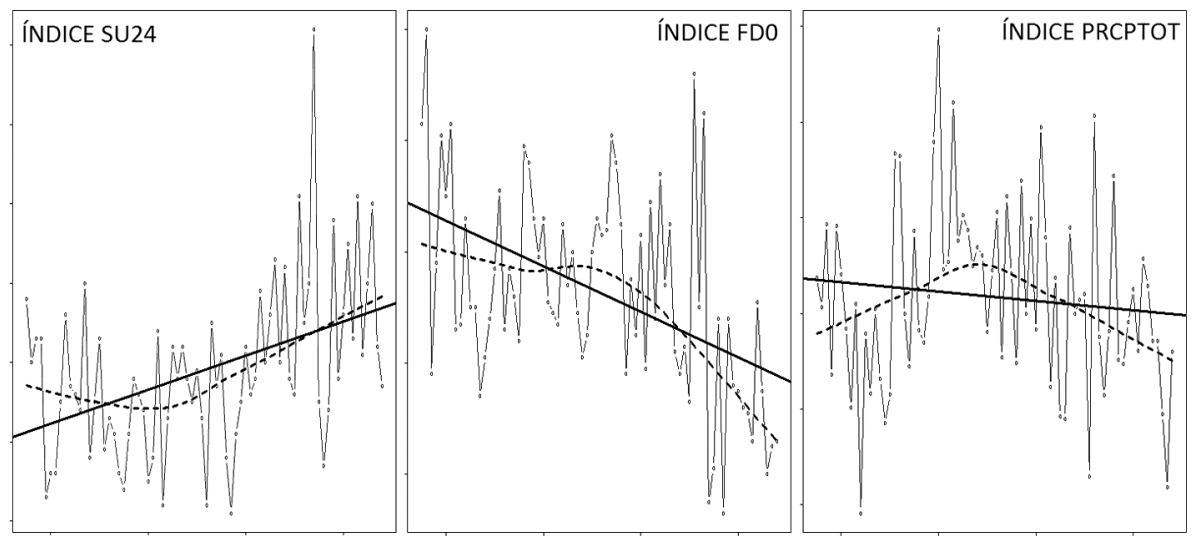

Figura 4. Evolución temporal de los índices SU24 (días con temperatura máxima superior a los $24^{\circ} \mathrm{C}$ ), FD0 (días con temperatura mínima inferior a $0^{\circ} \mathrm{C}$ - días de helada) y PRCPTOT (total de precipitación anual) para la estación de Ransol (1645m) y para el periodo 1935-2008.

Figure 4. Temporal evolution of the indexes SU24 (days with maximum temperature above $24^{\circ} \mathrm{C}$ ), FD0

(days with minimum temperature below $0{ }^{\circ} \mathrm{C}$ - days of frost) and PRCPTOT (total annual precipitation) for the station Ransol (1645m) and for the period 1935-2008.

decir, un mínimo de seis días consecutivos en los que la temperatura máxima supera el umbral del percentil 90) y en la amplitud térmica diaria anual.

En cuanto a los índices de precipitación, no se detecta ningún índice con tendencia significativa, siguiendo los resultados apreciados en el análisis de la tendencia estacional y anual. Tal vez pudiera destacarse el índice Rx5day (cantidad máxima de precipitación acumulada en 5 días consecutivos) que presenta una tendencia al descenso (aunque no significativo), al igual que el Prcptot (o precipitación total anual en los días húmedos, o con precipitación diaria superior a $1 \mathrm{~mm}$ ).

La figura 4 muestra gráficamente algunas de las tendencias en índices calculados para Ransol.

$\mathrm{Al}$ analizar el comportamiento de los mismos índices pero para el período 1950-2008, se pone en evidencia el incremento en aquellos índices térmicos sensibles al calentamiento (tabla 8). Así, además de los ya presentes en el análisis para todo el período disponible, aparecen nuevos índices con significación estadística en las tres series, tales como los llamados días de hielo, es decir, el número de días anuales en los que la temperatura máxima no supera los $0^{\circ} \mathrm{C}$ (o incluso umbrales más fríos) y que muestra un descenso apreciable. También la amplitud térmica anual presenta una tendencia positiva en todas las tres series, al igual que la duración de las rachas cálidas. 


\section{P. ESTEBAN VEA, M. PROHOM DURAN \& E. AGUILAR}

Tabla 8. Como en la tabla 7 pero para el periodo 1950-2008. En sombreado turquesa aquellos índices pluviométricos con significación estadística, según el valor de $\mathrm{p}(\mathrm{p}<0.05)$. Los índices su $24 / 26.2 / 28.1$, id $-2.1 / 0.9 / 1.5$, tr 8.3/13.1/12.4 y fd -10.6 / -7.3 / -5.9 se han calculado, respectivamente, con el valor medio de las máximas más altas para el mes más cálido (su), con valor medio de las máximas más baja para el mes más frio (id), con el valor medio de las mínimas más altas del mes más cálido (tr), y el valor medio de las mínimas más bajas del mes más frio (fd) según Raso (1999).

Table 8. As in Table 7 but for the period 1950-2008. Shaded in turquoise the rainfall rates with statistical significance, according to the value of $p<0.05$. The indices su $24 / 26.2 / 28.1, i d-2.1 / 0.9 / 1.5, \mathrm{tr}$ 8.3/13.1/12.4 and $f d-10.6 /-7.3 /-5.9$ are calculated, respectively, with the average of the highest maximum temperatures for the warmest month (su) with the average of the lower maximum temperatures for the coldest month (id), with the average of the highest minimum temperatures in the warmest month (tr) and the average of the lower minimum temperatures for the coldest month (fd) calculated in Raso (1999).

\begin{tabular}{|c|c|c|c|c|c|c|c|c|c|}
\hline \multirow{2}{*}{$\frac{1950-2008}{\text { Indices }}$} & \multicolumn{3}{|c|}{ RANSOL (1645m) } & \multicolumn{3}{|c|}{ ENGOLASTERS $(1640 \mathrm{~m})$} & \multicolumn{3}{|c|}{ CENTRAL $(1145 \mathrm{~m})$} \\
\hline & Pend. & $\begin{array}{l}\text { Desv. } \\
\text { Pend. }\end{array}$ & P_Valor & Pend. & $\begin{array}{l}\text { Desv. } \\
\text { Pend. }\end{array}$ & P_Valor & Pend. & $\begin{array}{l}\text { Desv. } \\
\text { Pend. }\end{array}$ & P_Valor \\
\hline su25 & 0.225 & 0.062 & 0.001 & 0.197 & 0.051 & 0 & 0.163 & 0.095 & 0.091 \\
\hline ido & -0.132 & 0.067 & 0.053 & -0.177 & 0.051 & 0.001 & -0.064 & 0.026 & 0.018 \\
\hline $\operatorname{tr} 20$ & 0 & 0 & $\mathrm{NaN}$ & 0 & 0 & $\mathrm{NaN}$ & 0 & 0 & $\mathrm{NaN}$ \\
\hline fdo & -0.535 & 0.117 & 0 & -0.325 & 0.146 & 0.03 & -0.401 & 0.11 & 0.001 \\
\hline su $24 / 26.2 / 28.1$ & 0.348 & 0.073 & 0 & 0.117 & 0.036 & 0.002 & 0.22 & 0.065 & 0.001 \\
\hline id $-2.1 / 0.9 / 1.5$ & -0.129 & 0.044 & 0.004 & -0.201 & 0.059 & 0.001 & -0.114 & 0.045 & 0.014 \\
\hline $\operatorname{tr} 8.3 / 13.1 / 12.4$ & 0.074 & 0.071 & 0.302 & 0.172 & 0.067 & 0.012 & 0.305 & 0.087 & 0.001 \\
\hline $\mathrm{fd}-10.6 /-7.3 /-5.9$ & -0.094 & 0.054 & 0.088 & -0.106 & 0.054 & 0.053 & -0.164 & 0.051 & 0.002 \\
\hline gsl & 0.378 & 0.217 & 0.087 & 0.259 & 0.266 & 0.334 & -0.023 & 0.221 & 0.919 \\
\hline txx & 0.049 & 0.013 & 0 & 0.041 & 0.014 & 0.004 & 0.038 & 0.013 & 0.004 \\
\hline txn & 0.028 & 0.019 & 0.157 & 0.028 & 0.021 & 0.182 & 0.027 & 0.019 & 0.149 \\
\hline $\operatorname{tn} x$ & 0.018 & 0.013 & 0.176 & 0.026 & 0.01 & 0.013 & 0.013 & 0.008 & 0.108 \\
\hline $\operatorname{tnn}$ & 0.058 & 0.018 & 0.002 & 0.04 & 0.019 & 0.041 & 0.061 & 0.016 & 0 \\
\hline tx10p & -0.035 & 0.019 & 0.077 & -0.071 & 0.021 & 0.001 & -0.049 & 0.024 & 0.043 \\
\hline tx $90 p$ & 0.181 & 0.03 & 0 & 0.14 & 0.027 & 0 & 0.182 & 0.027 & 0 \\
\hline $\operatorname{tn} 10 p$ & -0.075 & 0.023 & 0.002 & -0.036 & 0.026 & 0.164 & -0.036 & 0.026 & 0.169 \\
\hline $\operatorname{tn} 90 p$ & 0.08 & 0.032 & 0.016 & 0.094 & 0.032 & 0.004 & 0.133 & 0.044 & 0.004 \\
\hline wsdi & 0.223 & 0.079 & 0.007 & 0.154 & 0.045 & 0.001 & 0.251 & 0.061 & 0 \\
\hline $\operatorname{csdi}$ & -0.06 & 0.037 & 0.112 & 0.025 & 0.041 & 0.545 & 0.039 & 0.03 & 0.199 \\
\hline dtr & 0.014 & 0.003 & 0 & 0.014 & 0.003 & 0 & 0.012 & 0.003 & 0 \\
\hline rx1day & -0.062 & 0.157 & 0.692 & & & & -0.082 & 0.162 & 0.616 \\
\hline rx5 day & -0.35 & 0.245 & 0.158 & & & & -0.483 & 0.271 & 0.08 \\
\hline sdif & -0.005 & 0.008 & 0.58 & & & & -0.011 & 0.01 & 0.239 \\
\hline r10mm & -0.151 & 0.05 & 0.004 & & & & -0.147 & 0.053 & 0.008 \\
\hline $\mathrm{r} 20 \mathrm{~mm}$ & -0.118 & 0.03 & 0 & & & & -0.077 & 0.029 & 0.009 \\
\hline $\mathrm{r} 50 \mathrm{~mm}$ & -0.011 & 0.008 & 0.18 & & & & -0.008 & 0.01 & 0.454 \\
\hline odd & 0.044 & 0.063 & 0.48 & & & & 0.105 & 0.069 & 0.131 \\
\hline owd & -0.025 & 0.014 & 0.08 & & & & -0.019 & 0.01 & 0.064 \\
\hline r95p & -1.863 & 0.958 & 0.057 & & & & -0.506 & 0.894 & 0.574 \\
\hline r99p & -0.538 & 0.551 & 0.333 & & & & -0.17 & 0.516 & 0.743 \\
\hline preptot & -4.436 & 1.372 & 0.002 & & & & -3.293 & 1.452 & 0.027 \\
\hline
\end{tabular}


Pero la novedad más evidente que se detecta al centrar el análisis en el período 1950-2008 está en aquellos índices relativos a la precipitación diaria. Así, tanto en Ransol como en Central se aprecia un descenso significativo en tres índices: r10mm (número de días en un año en los que la precipitación diaria supera los $10 \mathrm{~mm}$ ), r20 $\mathrm{mm}$ (número de días en un año en los que la precipitación diaria supera los $20 \mathrm{~mm}$ ) y prcptot (precipitación total anual en los días húmedos, o con precipitación diaria superior a $1 \mathrm{~mm}$ ). Estos datos se asocian a una tendencia general hacia el descenso de la precipitación en el área de estudio.

\section{Discusión y conclusiones}

La disponibilidad en el Principado de Andorra de tres series climáticas con una amplia cobertura temporal y continuidad, permiten analizar con precisión la evolución reciente del clima en este particular área geográfica del Pirineo. A partir de un análisis previo en el que se ha puesto a prueba la calidad de los registros, y en el que se ha analizado su homogeneidad y corregido aquellos períodos no homogéneos utilizando los métodos actualmente más aceptados, se han generado series climáticas de calidad y fiables.

Así, para la variable temperatura y para el conjunto de las tres series estudiadas, la tendencia al incremento es patente y especialmente sólida para la temperatura máxima media anual (incremento de 0.13 a $0.15^{\circ} \mathrm{C} /$ década), y durante la estación estival (la temperatura media máxima estival se incrementa en $0.22^{\circ} \mathrm{C} /$ década, mientras que la temperatura media mínima estival lo hace en $0.11^{\circ} \mathrm{C} /$ década).

Considerando la tendencia acumulada, se puede concluir que la temperatura media anual en Andorra ha aumentado $0.7^{\circ} \mathrm{C}$ durante el período 19352008, unos valores que permiten afirmar que se ha constatado un calentamiento del clima en Andorra en los últimos 73 años. Esta afirmación no debería sorprender, ya que todos los estudios realizados en el área cercana (España, Pirineos, Francia) van en la misma línea.

En cuanto a la precipitación, para el período 1935-2008 no se aprecia una tendencia clara, aunque para la estación de Central se obtiene un descenso en la precipitación anual de 5.4\%/década, estadísticamente significativo. En cambio, si nos centramos en el periodo 1950-2008, los resultados son más contundentes tanto a nivel anual como, especialmente para el verano, que se muestra como la estación que de forma clara está notando un cambio en su comportamiento medio en relación al total de precipitación. 
Los resultados obtenidos tanto para la temperatura y la precipitación se ven reforzados cuando se obtienen los índices de cambio climático del ECCC basados a resolución diaria y que aportan más luz sobre el posible comportamiento de los extremos climáticos en el futuro. En las temperaturas, destacan para el periodo 1935-2008 las tendencias asociadas a las máximas (su25, txx y tx90p), unas tendencias que se consolidan o refuerzan para el periodo 1950-2008. En definitiva, los resultados de los índices también reflejan el calentamiento sufrido en las temperaturas ya observado con las tendencias anuales y estacionales, y especialmente en las máximas.

Para la precipitación, la falta de tendencias claras en el análisis estacional y anual para el periodo 1935-2008, también queda reflejado en los índices diarios con ninguna tendencia significativa, hecho que cambia sustancialmente cuando la ventana temporal se acota a 1950-2008, siendo un claro ejemplo el índice asociado a los totales anuales (prcptot), que refleja un descenso evidente. Un resultado similar ha sido encontrado para el noreste de la Península Ibérica (López-Moreno et al., 2010).

Es interesante hacer notar que las tendencias de temperatura y precipitación obtenidas están en consonancia con los resultados obtenidos para el conjunto de España (Agencia Estatal de Meteorología, Brunetet al., 2007), Noreste de la Península Ibérica (López-Moreno et al., 2010; El Kenawyet al., 2011), Cataluña (Boletín Anual de Indicadores Climáticos, ServeiMeteorológic de Catalunya, 2010) y Francia (Météo-France, Spagnoliet al., 2002; Maris et al., 2009). En futuros estudios, se abordarán otros aspectos de interés para el área de estudio, tales como la tendencia en los días de nevada y en los grosores del manto de nieve, variables que debido a su naturaleza requieren de un mayor estudio. Asimismo, deberá tratarse con especial atención el impacto que sobre la homogeneidad de los registros analizados tendrá el paso de observaciones manuales a observaciones automáticas, un cambio que se ha producido en las tres series recientemente.

\section{Agradecimientos}

A ForcesElèctriquesd'Andorra (FEDA), a Meteofrance, al proyecto COST ES0601-HOME, al proyecto CTP2010: Influència del Canvi_Climàtic en el Turisme de Neu al Pirineu, y al Grupo Climatología de la Universidad de Barcelona (2009 SGR 443). También a Natalia Gallego del CENMA, Meteofrance y al Dr. Olivier Mestre. 


\section{Referencias}

Aguilar, E., Auer, I., Brunet, M., Peterson, T. C. \& Wieringa, J., 2003. Guidelines on Climate Metadata and Homogenization.WCDMP-53, WMO-TD1186.Ginebra: World Meteorological Organization. 51 p.

Böhm, R.; Auer, I.; Schöner, W., Ganekind, M., Gruber, C., Jurkovic, A., Orlik, A. \& Ungersböck, M., 2009. Eine neue Webseite mit instrumentellen QualitätsKlimadaten für den Grossraum Alpen zurück bis 1760. Wiener Mitteilungen Band 216: Hochwässer: Bemessung, Risikoanalyse und Vorhersage

Brunet, M., Jones, P., Sigró, J., Saladié, O., Aguilar, E., Moberg, A., DellaMarta, P.M., Lister, D., Whalter, A. \& López, D., 2007. Temporal and spatial temperature variability and change over Spain during 1850-2005. Journal of Geophysical Research, 112: D12117, doi:10.1029/2006JD008249.

Brunet, M., Saladié, O., Jones, P., Sigró, J., Aguilar, E., Moberg, A., Lister, D., Walther, A. \&Almarza, C., 2008. A case-study/guidance on the development of long-term daily adjusted temperature datasets.WMO-TD-1425, WCDMP66.Ginebra: World Meteorological Organization. 43 p.

ServeiMeteorològic de Catalunya, 2010. Butlletí Anuald'Indicadors Climàtics. Any 2010.

Caussinus, H. \&Lyazrhiu, F., 1997. Choosing a linear model with a random number of change-points and outliers.Ann. Inst. Statist. Math., 49 (4): 761765.

Caussinus, H. \&Mestre, O. 2004. Detection and correction of artificial shifts in climate series. Journal of the Royal Statistical Society. Series C. Applied Statistics, 53: 405-425.

Diaz, HF., Grojean, M. \& Graumlich, L., 2003. Climate variability and change in high elevation regions: past, present and future. Climatic Change, 59: 1-4.

Domonkos, P., 2011. Adapted Caussinus-Mestre Algorithm for homogenising Networks of Temperature series (ACMANT), Int. J. Geosci., 2:293-309. doi:10.4236/ijg.2011.23032

Della-Marta, PM. \& Wanner H., 2006. A method of homogenizing the extremes and mean of daily temperature measurements. Journal of Climate, 19(17):4179-4197.

El-Kenawy, A., López-Moreno, J. I., Vicente-Serrano, S. M., 2011. Recent changes in daily temperature extremes in Northeastern Spain: 1960-2006. Natural Hazards and Earth System Sciences 11: 1-21.

Esteban, P., Prohom, M. J., Aguilar, E. \& Mestre, O., 2009. Evoluciórecent de la temperatura $i$ de la precipitació a Andorra (1934-2008): Resultatsanuals i estacionals. Andorra: CENMA (Centre d'Estudis de la Neu i de la Muntanyad'Andorra de l'Institutd'EstudisAndorrans). AND.757-2009. ISBN: 97899920-2-054-8. 
Klein Tank, A. M. G., Zwiers, F. W. \& Zhang, X., 2009. Guidelines on Analysis of extremes in a changing climate in support of informed decisions for adaptation. WMO-TD No. 1500, 56 pp.

Kuglitsch, F. G., Toreti, A., Xoplaki, E., Della-Marta, P. M., Luterbacher, J. \& Wanner, H., 2009. Homogenization of daily maximum temperature series in the Mediterranean Journal of Geophysical Research-Atmospheres 114: D15108, doi:10.1029/2008JD011606.

Maris, M., Giraud, G., Durand, Y., Navarre, J. P. \& Mérindol, L., 2009. Results of 50 years of climate reanalyses in the French Pyrenees (1958-2008) using the $S A F R A N$ and CROCUS models. Davos: International Snow Science Workshop, Proceedings.

Mestre, O., Gruber, C., Prieur, C., Caussinus; H. \& Jourdain, S., 2011. SPLIDHOM: A Method for Homogenization of Daily Temperature Observations. J. Appl. Meteor. Climatol., 50: 2343-2358. doi: 10.1175/2011JAMC2641.1

Nogués-Bravo, D., Araújo, M. B., Errea, M. P. \& Martínez-Rica, J. P., 2007. Exposure of global mountain systems to climate warming during the 21st century. Global Environmental Change, 17: 420-428.

Peterson, T. C. \& coauthors. 2001. Report on the Activities of the Working Group on Climate Change Detection and Related Rapporteurs 1998-2001. WMO, Rep. WCDMP-47, WMO-TD 1071, Geneve, Switzerland, 143pp.

Raso, JM., 1999. El clima d'Andorra. A Monografies de Geografia, Ministerid'Educació, Joventut i Esports del Governd'Andorra. 139 pp.

Spagnoli, B., Planton, S., Mestre, O., Déqué, M. \& Moisselin, J. M., 2002. Detecting climate change at a regional scale: the case of France. Geophys. Res. Lett., 29-10: 91-94.

Venema, V., Mestre, O., Aguilar, E., Auer, I., Guijarro, J. A., Domonkos, P., Vertacnik, G., Szentimrey, T., Stepanek, P., Zahradnicek, P., Viarre, J., Müller-Westermeier, G., Lakatos, M., Williams, C. N, Menne, M., Lindau, R., Rasol, D., Rustemeier, E., Kolokythas, K., Marinova, T., Andresen, L., Acquaotta, F., Fratianni, S., Cheval, S., Klancar, M., Brunetti, M., Gruber, CH., Prohom-Duran, M., Likso, T., Esteban, P. \& Brandsma, TH. 2011. Benchmarking monthly homogenization algorithms. Clim. Past Discuss., 7: 2655-2718.

Vincent, L. A., Zhang, X., Bonsal, B. R. \& Hogg, W. D., 2002. Homogenization of daily temperatures over Canada.J.Climate, 15: 1322-1334. 